\title{
Miniscrews: Contemporary Applications in Orthodontics
}

\author{
Elahe Soltanmohamadi Borujeni, and Homa Farhadifard ${ }^{1, *}$ \\ ${ }^{1}$ Department of Orthodontics, School of Dentistry, Tehran University of Medical Sciences, Tehran, IR Iran \\ *Corresponding author: Homa Farhadifard, Department of Orthodontics, School of Dentistry, Tehran University of Medical Sciences, Tehran, IR Iran. E-mail: homa.far1989@gmail.com
}

Received 2015 October 22; Accepted 2015 December 6.

\begin{abstract}
Context:The use of skeletal anchorage not only changed how far teeth can be moved, but also offered more treatment options to patients and clinicians. So many orthodontists use them in severe malocclusions to save patient from orthognatic surgery and the other invasive treatment plans. So our information about them must increase to use them in a right way.

Evidence Acquisition: To know recent findings about miniscrews and their use in orthodontic treatments

Results: In this review article, by focusing on the newest papers published in PubMed (from 2010 - 2015) we want to survey the most common applications of miniscrews in today orthodontics. To ease reading this paper, applications where divided into 7 groups. Almost all of papers used in this article had a common conclusion about safety and effectiveness of miniscrews in treatment of patients.

Conclusions: miniscrews are helpful in increasing treatment options for various malocclusions and they make treatment simpler, shorter and more comfortable for patients and clinicians.
\end{abstract}

Keywords: Orthodontic Anchorage Procedure, Molar, Dental Arch

\section{Context}

Nowadays skeletal anchorages (miniscrews and miniplates) are used in orthodontic treatment in a large-scale. The use of them not only changed how far teeth can be moved, but also offered more treatment options to patients and clinicians. So many orthodontists use them in severe malocclusions to save patient from orthognathic surgery and the other invasive treatment plans. According to a systematic review done by Papageorgiou in 2012, orthodontic miniscrew implants have a modest small mean failure rate, indicating their usefulness in clinical practice (1). But as we know use of skeletal anchorages have some risks and complications. Complications can arise during minscrew insertion or after orthodontic loading. A thorough understanding of placement technique, bone density, soft tissue, regional anatomic structures, and patient home care are important in success rate.

\section{Evidence Acquisition}

In this review article, by focusing on the newest papers published in PubMed (from 2010-2015) we want to survey the most common applications of miniscrews in today orthodontics. To ease reading this paper, applications where divided into 7 groups.

\subsection{Miniscrew as Anchorage Unit}

Anchorage control during tooth movement is one of the main factors for ensuring successful orthodontic treat- ment. The use of miniscrews to obtain absolute anchorage is so beneficial. In this way, the miniscrew is used in two ways: direct anchorage or indirect anchorage. As indirect anchorage, they are used to avoid undesirable movements of anchorage teeth, while conventional mechanics are used to retract anterior teeth. As direct anchorage, elastic chains are applied directly between the miniscrew placed between the second premolar, the first molar, and the hooks on the arch wire. Therefore, the point of force application is close to the center of resistance of the anterior teeth, so that the anterior segment may slide bodily with minimal tipping. In some articles, the use of conventional anchorage appliances like headgear was compared to miniscrews: Kuroda announced that both miniscrew and headgear gear anchorage were acceptable in overjet reduction and improvement of facial profile. However, miniscrew anchorage did not require patient cooperation to reinforce the anchorage and improve patient profile significantly. Therefore, he said that miniscrew anchorage is simpler and more useful than traditional anchorage for patients with Cl II malocclusion (2). Koyama in 2011 found that in comparison to straight-pull headgear and intermaxillary elastics, sliding mechanics with implant anchorage provide absolute anchorage and could control mandibular rotation better (3). Lee and others in 2013 published an RCT article about the effects of midpalatal miniscrews versus headgear for anteroposterior and vertical anchorage in patients with $\mathrm{Cl}$ II malocclusion. The results reveal that miniscrew group has less mesial movement of

Copyright (C) 2015, Iranian Journal of Orthodontics. This is an open-access article distributed under the terms of the Creative Commons Attribution-NonCommercial 4.0 International License (http://creativecommons.org/licenses/by-nc/4.0/) which permits copy and redistribute the material just in noncommercial usages, provided the original work is properly cited. 
the first maxillary molars and greater maxillary incisors retraction. Maxillary molars were significantly intruded in miniscrew group so mandibular plan angle was decreased significantly, but they were extruded in the headgear group. They concluded that in both anteroposterior and vertical directions, a modified transpalatal arch supported by 2 miniscrews provided more stable anchorage (4). In the other study, Lee in 2014 compared treatment duration and dentoskeletal changes in 2 different anchorage systems used to treat maxillary dentoalveolar protrusion and examined the effectiveness of an enmasse retraction with 2 miniscrews in midpalatal suture. They found that by using miniscrews orthodontists have more time, control the anterior teeth during enmasse retraction (approximately 4 months) without increasing total treatment duration. With miniscrews, better dentoskeletal control (greater change in ANB angle) can be achieved so the quality of treatment can totally improve with them (5). Effectiveness of miniscrews and miniplates for treatment of protrusive maxillary dentition was also investigated with 3D model analysis. Analysis showed significant differences (greater anterior retraction, less molar protraction) between headgear and mini-implant groups (6).

\subsection{Molar Distalization}

During molar distalization with conventional intraoral appliances, tipping and extrusion can occur in conjunction with the distal movement. In addition, reactive forces on the anterior anchoring teeth occur. Many types and designs of appliances such as the pendulum, distal-jet and the compressed coil spring can be combined with a miniscrew anchorage system. For bilateral molar distalization, rotation, tipping, and extrusion can be controlled by placing the miniscrews in both the buccal and palatal region, and by using transpalatal arches. Chung 2010, announced that molar distalization with the combination of osteointegrated mini implants, segmented arch wires, minimum bonded attachments is a simple and effective treatment choice in a distalization treatment. He corrected unilateral Cl II malocclusions with this method (7). Mariani et al. in 2014 investigated dentoalveolar and skeletal effects of 2 anchorage systems for treatment of $\mathrm{Cl}$ II malocclusions: skeletal anchorage and Pendulum (intraoral anchorage). They found that inskeletal anchorage group the upper molar distalization was achieved faster and greater. Anterior anchorage loss occurred in both groups, although in skeletal anchorage group, there was less mesial movement of premolars. Finally they concluded that both appliances are effective in the correction of class II malocclusion but skeletal anchorage group is more efficient, producing greater molar distalization in a shorter time (8).

\subsection{Molar Uprighting}

Uprighting vectors with intrusion are very hard to accomplish; therefore, absolute anchorage is required.
Miniscrews can be used as direct anchorage to prevent reactive forces on adjacent teeth that may result in negative side effects during tooth uprighting. Cernei in 2015 in a case report searched the effects of using miniscrew in 2nd molar uprighting after premature loss of mandibular first molar. According to his idea, correction of vertical problems has become easier with miniscrews. He inserted a miniscrew between canine and first premolar. Uprighting occurred without unwanted movements in anterior mandibular region and without molar extrusion. Therefore, he announced that using miniscrews in such cases decrease unwanted side effects and improve quality of treatment (9).

\subsection{Force Eruption}

Miniscrews represent a step towards resolving the problem of anchorage in orthodontics, including in forced eruption cases. Miniscrews for skeletal anchorage proved to be an effective device to obtain extrusion for prosthetic purposes without undesired movements on other teeth and with no need for patient compliance when used in the same arch (10). In 2 other papers, forced eruption of impacted teeth by bracket head miniscrew were discussed $(11,12)$.

\subsection{Intrusion}

\subsubsection{Intrusion of Anterior Teeth}

Miniscrews may be used to stabilize the molars during the incisor intrusion process, or can be placed anteriorly and used for direct application of the intrusive force to the incisors. The miniscrews should be placed as close to the midline of the anterior arch as possible. Alternately two miniscrews may be inserted into the lateral and canine interradicular area on both left and right sides. To compare the clinical effects of miniscrews and conventional utility arch in the maxillary incisors intrusion, Ma in 2013 in a RCT paper reported that in miniscrew group there was no change in U1-SN \& U6-PP distance. Probing depth was increased and the width of keratinized gingiva decreased significantly. He concluded that miniscrew has advantages on intruding upper incisors compared with conventional utility arch and improving tooth-lip relationships. Less treatment period is needed in miniscrew group and the impact on periodontal supporting tissues between 2 groups was similar (13). Kalra in 2015 published an article about miniscrew supported Burstone intrusion arch for achieving maxillary incisor intrusion. He introduced a modification of intrusion arch that take posterior anchorage from buccual interradicular miniscrews instead of molar teeth. Finally he concluded that this method have some advantages over conventional intrusion arch (14). Ishihara treated deep overbite in a patient with cl II malocclusion by sectional arch wires and miniscrews placed in the premolar areas. The resultant occlusion and satisfactory facial profile remain after 30 months (15). Kim in 2014 reported 
a case with $\mathrm{Cl}$ II/div II malocclusion and gummy smile treated by miniscrew and continuous arch with additional torque for intrusion, retraction, and torque control of upper incisors. The results were intrusion and lingual root movement of maxillary incisors without anchorage loss of maxillary molars, flattening of the curve of Spee and class I molar relation that were maintained after 50 months of retention period. Therefore, he concluded that combined use of minscrew and continuous arch could be a reliable and effective treatment modality for torque control and intrusion of retroclined maxillary incisors in $\mathrm{Cl}$ II/div 2 malocclusions (16).

\subsubsection{Intrusion of Posterior Teeth}

Anterior open bites can be closed successfully through the intrusion of posterior teeth using various mechanical methods incorporating miniscrews. Intrusion of posterior teeth is considered one of the most difficult types of tooth movement to achieve using conventional mechanics. miniscrew-combined treatment may solve this problem.

Cao in 2013 introduced a new treatment method using palatal fixed edgewise appliances and miniscrews to intrude the overerupted upper second molars. Clinical results showed a significant intrusion effect without root resorbtion and periodontal problems. So it is a simple and effective way to intrude second molars especially in situations where miniscrews can not be inserted directly next to the second molar (17). according to Yanagita in 2013 and after treatment a case of severe open bite due to traumatic condylar fracture non surgically, Although there was some relapse, results suggest that implant anchorage is useful for correcting anterior open bites originating from condylar fractures (18).

\subsubsection{Molar Protraction}

Protraction of mandibular molars is challenging because of the high density of mandibular bone. Anterior dental anchorage is often inadequate to protract even a single first molar without reciprocal retraction of the incisors or movement of the dental midline. Using miniscrews for mandibular molar protraction, avoiding often encountered with the use of dental anchorage. In a RCT paper by Kim in 2015, mandibular molar protraction as an alternative treatment for edentulous spaces was investigated. External apical root resorbtion and alveolar bone loss after protraction were measured and results were announced. Fifty one mandibular molars were protracted by using sliding mechanics with a lever arm or a miniscrew-supported root spring. All spaces closed successfully. Root length significantly decreased $(0.8 \mathrm{~mm})$ but root resorbtion more than $2 \mathrm{~mm}$ occurred in only $4 \%$ of molars. Alveolar bone height reduced significantly by $0.56 \mathrm{~mm}$ but alveolar bone loss more than $2 \mathrm{~mm}$ occurred in only $2 \%$ of molars. He concluded that this method for molar protraction is so safe and effective especially in young adults (19). In the other case report by Baik in 2012, 2nd molar protraction to close space of extracted first molar was done. Simultane- ously, closing anterior open bite by intrusion of maxillary molars and extrusion of anterior teeth, was done. One year after treatment, spaces of extracted teeth were closed and Mandibular 3rd molar erupted in the space of 2nd molar successfully. Open bite became so better in comparison to beginning of treatment (20).

\subsubsection{Midface Protraction}

Tooth-anchored facemask therapy is a common protocol for treatment of class III malocclusions with midface deficiency. But it has some adverse effects, such as extrusion and mesialization of maxillary molars. Use of skeletal anchorage may be useful to avoid these effects. Nevertheless, as we use this treatment in mixed dentition period, position of permanent teeth in jawbone is so important to avoid hurt them. To evaluate the effectiveness of TAD anchored maxillary protraction in late mixed dentition, Feng in 2012, gathered RCTs and after meta-analysis announced that TAD-anchored maxillary protraction have a greater maxillary advancement effect and might reduce skeletal and dental side effects, compared with tooth-anchored maxillary protraction (21). Solano-Mendoza searched different types of early treatment cl III malocclusions with maxillary origin. He concluded that miniplates and miniscrews offer a purely orthopedic approach to treatment with minimization of the undesirable side effects of traditional dentofacial orthopedic compensation based on the dentoalveolar anchorage (22). In a case report, Cha found that maxillary protraction can be done by skeletal anchorage with better control and without other tooth support (23).

\section{Results}

Almost all of papers used in this article had a common conclusion about safety and effectiveness of miniscrews in treatment of patients. Although they have some complications during insertion or force application but they can facilitate treatment and help orthodontists to save their time and increase the quality of treatment.

\section{Conclusions}

Today, patient's expectations from orthodontic treatment are increased. They want the best results including lower time consume, better esthetics and using methods less invasive and with less cooperation. Mini-implants (miniscrews and miniplates) that are so familiar for every orthodontist can be helpful to reach treatment goals with less complications. So based on this fact that they are widely used in daily practice, there are so many papers available about their features, complications, risk factors, and applications. By focusing on Pubmed, we found many articles about recent use of miniscrews that some of them were discussed above. We generally knew that according to scientific bases, miniscrews are help- 
ful in increasing treatment options for various malocclusions and they make treatment simpler, shorter, and more comfortable for patients and clinicians

\section{Acknowledgments}

The authors wish to thank professor Allahyar Geramy for his guide and help in preparing this paper.

\section{Footnote}

Authors' Contribution:Elahe Soltanmohamadi Borujeni: data gathering, and collection; Homa Farhadifard: writing the main part.

\section{References}

1. Papageorgiou SN, Zogakis IP, Papadopoulos MA. Failure rates and associated risk factors of orthodontic miniscrew implants: A meta-analysis. Am J Orthod Dentofacial Orthop. 2012;142(5):577-595 e7. doi:10.1016/j.ajodo.2012.05.016. [PubMed: 23116500]

2. Kuroda S, Yamada K, Deguchi T, Kyung HM, Takano-Yamamoto T. Class II malocclusion treated with miniscrew anchorage: Comparison with traditional orthodontic mechanics outcomes. Am J Orthod. 2009;135(3):302-9. doi:10.1016/j.ajodo.2007.03.038.

3. Koyama I, Iino S, Abe Y, Takano-Yamamoto T, Miyawaki S. Differences between sliding mechanics with implant anchorage and straight-pull headgear and intermaxillary elastics in adults with bimaxillary protrusion. Eur J Orthod. 2011;33(2):126-31. doi: 10.1093/ejo/cjq047. [PubMed: 21059875]

4. Lee J, Miyazawa K, Tabuchi M, Kawaguchi M, Shibata M, Goto S. Midpalatal miniscrews and high-pull headgear for anteroposterior and vertical anchorage control: Cephalometric comparisons of treatment changes. Am J Orthod. 2013;144(2):238-50. doi: 10.1016/j.ajodo.2013.03.020

5. Lee J, Miyazawa K, Tabuchi M, Sato T, Kawaguchi M, Goto S. Effectiveness ofen-masseretraction using midpalatal miniscrews and a modified transpalatal arch: Treatment duration and dentoskeletal changes. KJO. 2014;44(2):88. doi: 10.4041/kjod.2014.44.2.88. [PubMed: 24696825]

6. Lai EHH, Yao CCJ, Chang JZC, Chen I, Chen YJ. Three-dimensional dental model analysis of treatment outcomes for protrusive maxillary dentition: Comparison of headgear, miniscrew, and miniplate skeletal anchorage. Am J Orthod. 2008;134(5):636-45. doi:10.1016/j.ajodo.2007.05.017.

7. Chung KR, Kim SH, Chaffee MP, Nelson G. Molar distalization with a partially integrated mini-implant to correct unilateral Class II malocclusion. Am J Orthod. 2010;138(6):810-9. doi: 10.1016/j.ajodo.2008.07.027.

8. Mariani L, Maino G, Caprioglio A. Skeletal versus conventional intraoral anchorage for the treatment of class II malocclusion: Dentoalveolar and skeletal effects. Progress in Orthodontics. 2014;15(1):1-10. doi: 10.1186/s40510-014-0043-Z. [PubMed: 24393493]

9. Cernei ER, Mavru RB, Zetui IN. Second molar uprighting after premature loss of mandibular first permanent molar-Case report. Rev Med Chir. 2014;119(2):572-8.

10. Derton N, Derton R, Perini A. Egression forcee avec mini vis Protocoles avec elastiques verticaux inter-arcade ou avec la technique intra-arcade de Derton-Perini. Deux cas cliniques. International Orthodontics. 2011;9(2):179-95. doi: 10.1016/j.ortho.2011.03.012. [PubMed: 21511550]

11. Kim JW, Park JY, Baek SH, Kim TW, Chang YI. Forced eruption of an impacted third molar using a bracket-head miniscrew. J Clin Orthod. 2010;44(5):313-8. [PubMed:20831100]

12. Heravi F, Shafaee H, Forouzanfar A, Zarch SH, Merati M. Forced eruption of palatally impacted canines using bracket-head miniscrews. J Clin Orthod. 2014;48(9):576-80. [PubMed:25329631]

13. Ma D, Wang XX, Jin SM, Dong R, Liu WX, Li J, et al. Comparison of two treatment method for maxillary incisors intrusion. Shanghai Kouqiang Yixue. 2013;22(2):206-9. [PubMed: 23708038]

14. Kalra S, Tripathi T. Miniscrew supported Burstone intrusion arch Int J Orthod Milwaukee. 2015;26(1):25-6. [PubMed: 25881380]

15. Ishihara Y, Kuroda S, Sugawara Y, Balam TA, Takano-Yamamoto T, Yamashiro T. Indirect usage of miniscrew anchorage to intrude overerupted mandibular incisors in a Class II patient with a deep overbite. Am JOrthod Dentofacial Orthop. 2013;143(4 Suppl):S113-24. doi:10.1016/j.ajodo.2012.09.001. [PubMed: 23540627]

16. Kim SJ, Kim JW, Choi TH, Lee KJ. Combined use of miniscrews and continuous arch for intrusive root movement of incisors in Class II division 2 with gummy smile. Angle Orthod. 2014;84(5):910-8. doi:10.2319/080713-587.1. [PubMed: 24512532]

17. Cao Y, Liu C, Wang C, Yang X, Duan P, Xu C. A simple way to intrude overerupted upper second molars with miniscrews.J Prosthodont. 2013;22(8):597-602. doi:10.1111/jopr.12057. [PubMed: 23725052]

18. Yanagita T, Adachi R, Kamioka H, Yamashiro T. Severe open bite due to traumatic condylar fractures treated nonsurgically with implanted miniscrew anchorage. Am J Orthod Dentofacial Or thop. 2013;143(4 Suppl):S137-47. doi: 10.1016/j.ajodo.2012.04.024. [PubMed: 23540631]

19. Kim SJ, Sung EH, Kim JW, Baik HS, Lee KJ. Mandibular molar protraction as an alternative treatment for edentulous spaces: Focus on changes in root length and alveolar bone height. JAm Dent AsSoc. 2015;146(11):820-9. doi: 10.1016/j.adaj.2015.04.025. [PubMed: 26514887]

20. Baik UB, Chun YS, Jung MH, Sugawara J. Protraction of mandibular second and third molars into missing first molar spaces for a patient with an anterior open bite and anterior spacing. Am J Orthod Dentofacial Orthop. 2012;141(6):783-95. doi: 10.1016/j.ajodo.2010.07.031. [PubMed:22640680]

21. Feng X, Li J, Li Y, Zhao Z, Zhao S, Wang J. Effectiveness of TADanchored maxillary protraction in late mixed dentition. Angle Orthod. 2012;82(6):1107-14. doi: 10.2319/111411-705.1. [PubMed: 22458766]

22. Solano-Mendoza B, Iglesias-Linares A, Yañez-Vico RM, MendozaMendoza A, Alió-Sanz JJ, Solano-Reina E. Maxillary protraction at early ages. The revolution of new bone anchorage appliances. $J$ Pedi Clinc Dent. 2012;37(2):219-29.

23. Cha BK, Choi DS, Ngan P, Jost-Brinkmann PG, Kim SM, Jang IS. Maxillary protraction with miniplates providing skeletal anchorage in a growing Class III patient. Am J Orthod Dentofacial Orthop. 2011;139(1):99-112. doi: 10.1016/j.ajodo.2009.06.025. [PubMed: 21195283] 